\title{
Analysis of the characteristics of patients admitted to Internal Medicine wards for exacerbation of chronic obstructive pulmonary disease, and discharge phase optimization. The SDO-ARCA Project of the Scientific Society FADOI
}

\author{
Marco Candela, ${ }^{1}$ Claudio Norbiato, ${ }^{2}$ Mauro Campanini, ${ }^{3}$ Antonio Luca Brucato, ${ }^{4}$ Salvatore Di Simone, ${ }^{5}$ \\ Dario Manfellotto, ${ }^{6}$ Francesco Dentali, ${ }^{7}$ Antonio Sacchetta, ${ }^{8}$ Antonella Valerio, ${ }^{9}$ Andrea Fontanella, ${ }^{10}$ \\ for the SDO-ARCA Study Group*
}

Correspondence: Antonella Valerio, Research Department, FADOI Foundation, Piazzale Cadorna 15, 20123 Milan, Italy. Tel.: +39.02.48005140 - Fax: +39.02.93662609.

E-mail: antonella.valerio@fadoi.org

Key words: Chronic obstructive pulmonary disease (COPD); Internal Medicine; GOLD class; hospital discharge phase.

Acknowledgments: we are grateful to Davide Ghilardi for the organization and start-up procedures and to all physicians and nurses involved in the daily activity at the participating centers.

Funding: the study was funded by FADOI Foundation, a notfor-profit organization (Italian Scientific Society of Hospital Internal Medicine). Authors express their gratitude to the Company GlaxoSmithKline S.p.A. for supporting the study with a research grant, without involvement in study design, execution, data collection, the decision to publish, or preparation of the manuscript.

Contributions: all authors participated in manuscript preparation and approved it.

Conflict of interests: all the authors Marco Candela, Claudio Norbiato, Mauro Campanini, Antonio Luca Brucato, Salvatore Di Simone, Dario Manfellotto, Francesco Dentali, Antonio Sacchetta, Antonella Valerio, Andrea Fontanella for the SDOARCA Study Group have declared no conflict of interests concerning the submitted manuscript.

Availability of data and materials: the data sets compiled and analyzed for the current study are available from the corresponding author on reasonable request.

Ethics approval and consent to participate: the study project was approved by the Ethics Committee of reference for the coordinating site (Marche Region Ethics Committee) and subsequently by the corresponding Ethics Committees of all the participating sites. Each patient gave his/her informed consent for participation in the study and to use their data for research purposes.

Received for publication: 26 May 2020.

Revision received: 8 June 2020.

Accepted for publication: 8 June 2020.

This work is licensed under a Creative Commons Attribution NonCommercial 4.0 License (CC BY-NC 4.0).

${ }^{\circ}$ Copyright: the Author(s), 2020

Licensee PAGEPress, Italy

Italian Journal of Medicine 2020; 14:156-161

doi:10.4081/itjm.2020.1321
${ }^{1}$ Medical Department, Hospital of Jesi and Area Vasta 2 ASUR Marche, Ancona; ${ }^{2}$ Department of Internal Medicine, Hospital Mauriziano, Torino; ${ }^{3}$ Department of Internal Medicine, Hospital Maggiore della Carità, Novara; ${ }^{4}$ Department of Internal Medicine, Hospital Papa Giovanni XXIII, Bergamo/Department of Internal Medicine, Fatebenefratelli, Milan; ${ }^{5}$ Department of Internal Medicine, Hospital of Colleferro, Rome; ${ }^{6}$ Department of Internal Medicine, Hospital Fatebenefratelli-AFaR, Isola Tiberina, Rome; ${ }^{7}$ Internal Medicine, Hospital Circolo Macchi, Varese/Internal Medicine, Hospital of Luino, ASST-Sette Laghi, and University of Insubria, Varese; ${ }^{8}$ Department of Internal Medicine, Hospital of Conegliano Veneto, Treviso; ${ }^{9}$ Research Department, FADOI Foundation, Milan; ${ }^{10}$ Medical Department, Hospital Buon Consiglio-Fatebenefratelli, Naples, Italy

* See Appendix II for the list of Sites and Investigators

\begin{abstract}
The purpose of this study was to have a nationwide snapshot of the characteristics of patients hospitalized in Internal Medicine Units (IMUs) for exacerbation of chronic obstructive pulmonary disease (COPD), and to assess applicability and contents of a specific Hospital Discharge Form for the patient with exacerbation of COPD discharged from IMUs. This was a prospective study in 44 IMUs in Italy, enrolling patients hospitalized with a diagnosis of COPD exacerbation. Information concerning clinical characteristics of patients, and treatment for COPD at discharge was collected. Specific documents for monitoring of clinical conditions and adherence to therapies as well as a form including individual indications for clinical controls, instrumental tests, etc. were provided upon discharge. Four hundred and seventy-two patients were enrolled ( $68 \%$ male). According to GOLD classification 2015, patients with classes A to D were $12 \%, 27 \%$, $31 \%$, and $30 \%$, respectively. Triple therapy was prescribed in $14 \%$ of GOLD A and $51 \%$ of GOLD D patients. Around $10 \%$ of patients for each GOLD class received no specific therapy. The vast majority of patients (85\%) received instructions on the correct use of inhalers, and in most cases $(85 \%)$, the quality of counseling was considered optimal/adequate. Indication for performing chest X-ray, spirometry, or blood gas analysis following discharge was addressed to $29 \%, 59 \%$ and $52.1 \%$ of patients, respectively. The follow-up sheet for COPD used in our study was shown as applicable. This highlighted the need for greater awareness and more standardized procedures within IMUs in the post-discharge phase.
\end{abstract}




\section{Introduction}

Chronic obstructive pulmonary disease (COPD) is the most common chronic lung disease, reaching a prevalence of $20 \%$ in people over the age of 70 and currently being the fourth most important cause of death worldwide ${ }^{1}$ and on target to become the third by 2020. COPD is also one of the biggest causes of morbidity, and the costs related to this disease are expected to increase globally due to progressive exposure to risk factors and ageing population. ${ }^{2}$

In the hospital setting, and in particular, in Internal Medicine Units (IMUs), COPD is one of the most common diseases encountered, both as a primary diagnosis for hospitalization and in cases with multiple concomitant diseases. ${ }^{3}$ The presence of this disease should be checked for in all people exposed to risk factors (smoking, occupational exposure, etc.) and with symptoms such as shortness of breath at rest or under stress, productive cough, and frequent bronchitis in their histories, even if they have other diagnoses at admission. ${ }^{4}$

Given the above, there is a need for increased awareness and accountability of the internist and/or the identification of specific training pathways that result in better engagement in COPD management, as a chronic disease with frequent flare-ups, and thus deserving particular and competent attention.

It is well established that optimal management of COPD in hospitalized patients requires specific experience and management expertise during hospitalization in order to better address and overcome the patient's acute/critical phase. However, it is also well known that discharge is a critical and crucial step to achieving one of the main goals in COPD management: a reduction of exacerbation. ${ }^{5}$ Exacerbations are accompanied by a progressive decline in respiratory function and frequent re-hospitalizations, which represent a significant social burden, amongst other factors. Taking appropriate measures that support discharge protection can allow a better self-management by the patient (or caregivers) at home $\mathrm{e}^{6-8}$ and, in terms of integration between the local health service of primary care and the hospital, the implementation of appropriate monitoring and follow-up strategies.

In recent years, the Italian Scientific Society of Internal Medicine (FADOI) has dedicated significant resources to the training of Internists, for proper management of patients with COPD. In keeping with the idea that the time of discharge is a key moment in initiating a good chronic patient management process, a few years ago the Scientific Society proposed a template of Hospital Discharge Form specifically designed for patients with COPD. ${ }^{9}$ The proposal to use this Form is based on the assumption that there are some documented conceptual and operational boundaries in the management of COPD, such as a prevalent focus on the acute phase of the disease, limited adoption of specific management tools, with the consequent risks of inadequate discharges, heterogeneous behavior between Centers, modest sensitivity to controls on therapeutic adherence, and poor communication with primary care health services. In this regard, we find that increased awareness among patients and their families/caregivers, and better involvement of primary care facilities could be facilitated by a structured and personalized transmission of information aimed at optimizing patient's therapy and monitoring.

With the study described in this paper, the objectives of FADOI were: i) to provide an updated picture of the main characteristics of patients admitted to Internal Medicine for COPD exacerbation, the diagnostic plans, and treatments used; ii) to focus on the point of discharge, systematically collecting (through a Hospital Discharge Form, a checklist and follow-up sheet) any indications that the physician wishes to transmit to the local health service and to the patient/caregiver to facilitate the patient's continuity of care and selfmanagement behaviors.

\section{Materials and Methods}

The SDO-ARCA (SDO is the acronym that qualifies, in Italy, the form for hospital discharge - ARCA acronym for Active listening, scientific Rigor, Connectivity and Adherence to therapy) study involved 44 Internal Medicine Units in Italy, in the period January 2016 - December 2017.

Consecutive patients admitted with a diagnosis of COPD exacerbation were included in the study, who were discharged from the IMUs and gave their informed consent to participate in the project. No exclusion criteria were set, except for patients with cognitive impairment unable to follow inhaled therapy correctly and, more generally, to comply with monitoring/follow-up guidelines provided in COPD-specific discharge documents.

With regard to the period of hospitalization, information was collected for each patient, such as age, gender, smoking habits, present comorbidities, known diagnosis or first diagnosis, history of exacerbations, clinical presentation at admission, functional, instrumental, and laboratory disease activity parameters (including natriuretic peptides and eosinophils), the possible execution of specific instrumental tests (e.g. spirometry, echocardiogram, etc.), drug and non-drug therapies, and COPD therapy at discharge.

The discharge checklist used in the study met the appropriateness criteria for discharge reported in the literature, as well as information on the use and quality of inhaled therapy, smoking habits and vaccinations, clinical monitoring scores such as COPD Assessment Test and St. George's Respiratory Questionnaire, ac- 
tivation of home care, and delivery of self-service information booklet. The follow-up sheet included the modalities of clinical-instrumental monitoring, the detection of the number of exacerbations, and the deadlines of subsequent observations, as well as any comorbidities present.

This documentation was completed by the Physician electronically and delivered to the patient in paper form, to monitor the diagnostic-therapeutic path, and to be passed on to the General Practitioner as well. For each patient, depending on the specific clinical needs, the attending physician who completed the Hospital Discharge Form also determined the follow-up period by which the proper specialist and diagnostic examinations should be performed, as well as the methods for monitoring the adherence to treatment prescriptions, and the possible occurrence of any major clinical events. The choice of follow-up duration, as well as of diagnostic and treatment program, was left to the judgment of the Internist who discharged the patient following the best medical practice for the individual patient, and not based on standardized study-specific programs.

Due to the descriptive and exploratory aim of the project, no formal calculation of the sample size was planned. Categorical variables were expressed as absolute number and percentage and continuous variables as mean and standard deviation or median and interquartile range, depending on the normal or nonnormal distribution of data.

The study is a not-for-profit project, sponsored by the Italian Scientific Society of Hospital Internal Medicine FADOI, and was conducted in accordance with Good Clinical Practices for Clinical Research and Regulations in Italy. In particular, the study project was approved by the Ethics Committee of reference for the coordinating site (Marche Region Ethics Committee) and subsequently by the corresponding Ethics Committees of all the participating sites. Each patient has given his/her informed consent for participation in the study and to use their data for research purposes. In order to assure subject confidentiality, data were pseudo-anonymized by means of a patient identification number and avoiding details such as date of birth and address. The link between patient study identification and information collected for the registry was maintained at the study sites only, and therefore unavailable for the staff of the Sponsor.

\section{Results}

Overall, 472 patients were enrolled in the study (median 11 for each site, range 1-29). Table 1 shows the main general patient's characteristics.

Figure 1 shows the distribution of COPD treatments at discharge for the different classes established by the GOLD 2015 stratification.
At the time of hospital discharge, 401 patients $(85 \%)$ had received instructions on the correct use of inhalers. In $27 \%$ of these patients, the quality of counseling and teach-back detection modes were optimal, adequate in $58 \%$, and inadequate in $15 \%$.

Table 2 presents the main indications in the follow-up sheet regarding general management and the type of controls expected after patient discharge.

Table 1. General characteristics of the patients enrolled in the study.

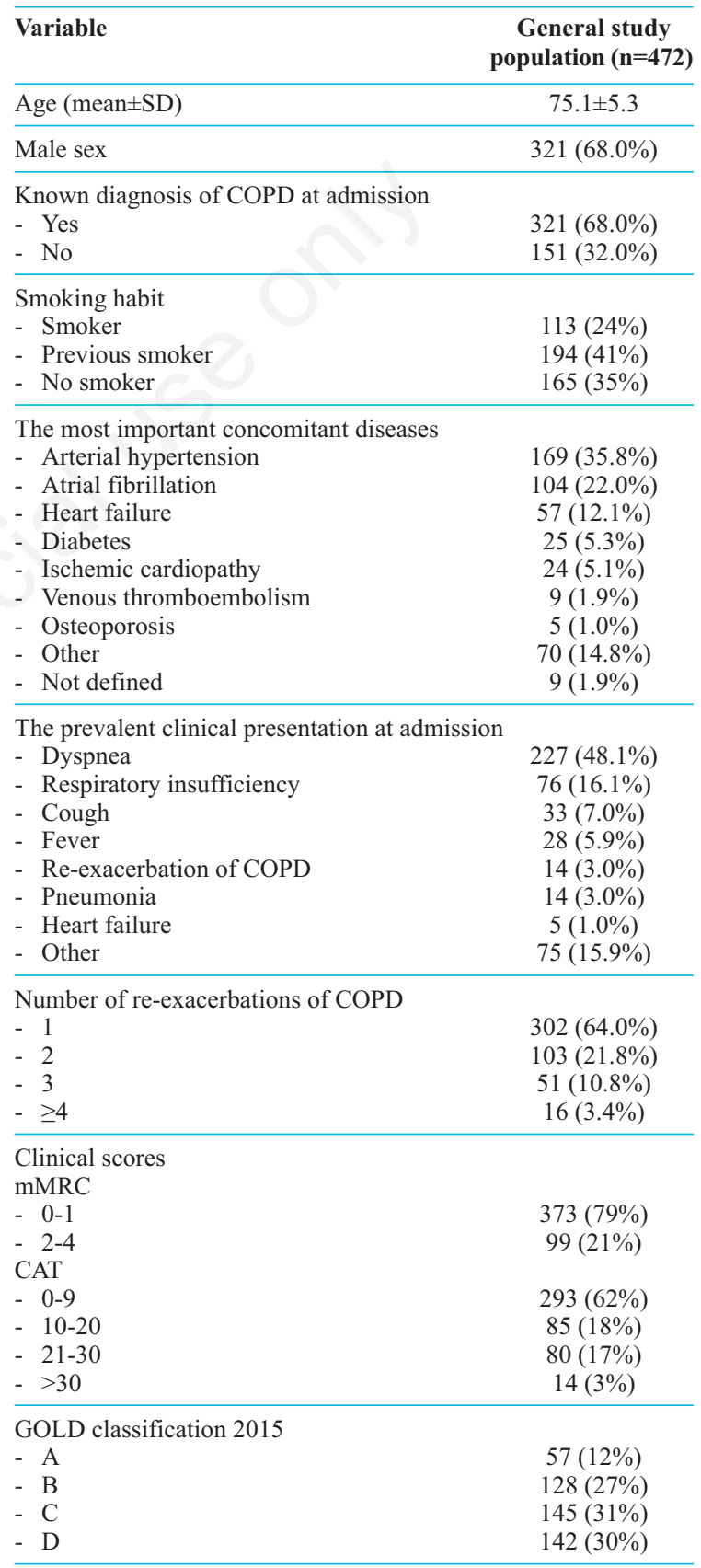

$\mathrm{SD}$, standard deviation; COPD, chronic obstructive pulmonary disease; mMRC, Modified British Medical Research Council Questionnaire; CAT, COPD Assessment Test [None, score <5; Low, score 5-9; Medium, score 10-20; High, score 21-30; Very High, score $>30]$. 


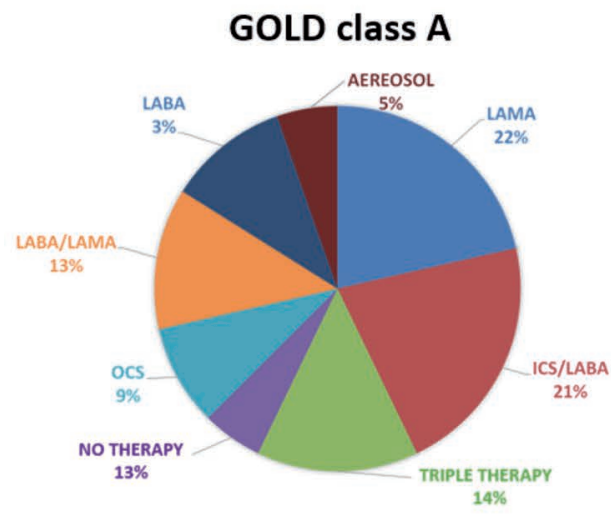

GOLD class B

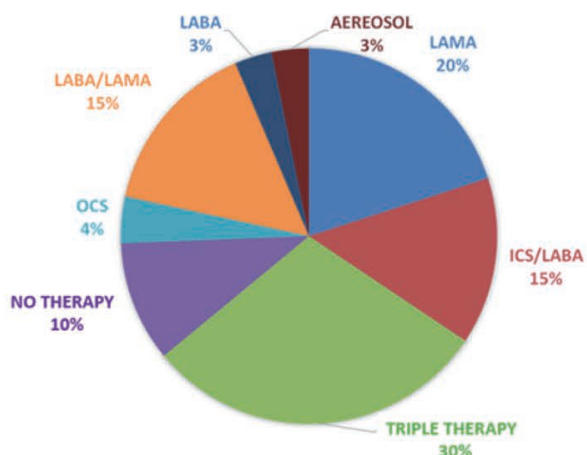

$30 \%$

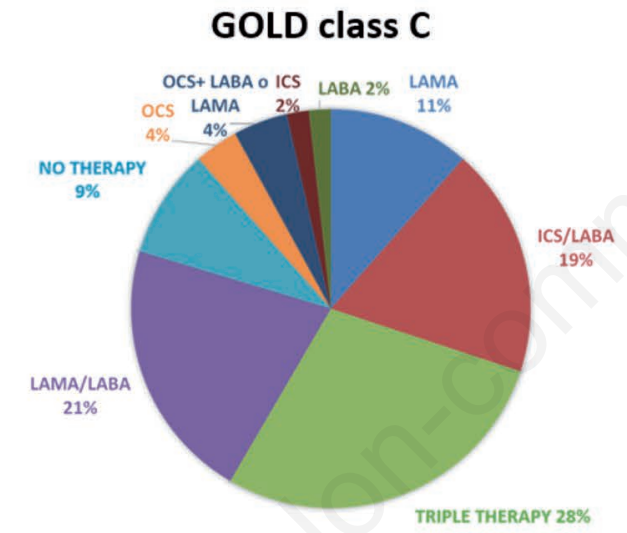

GOLD class D

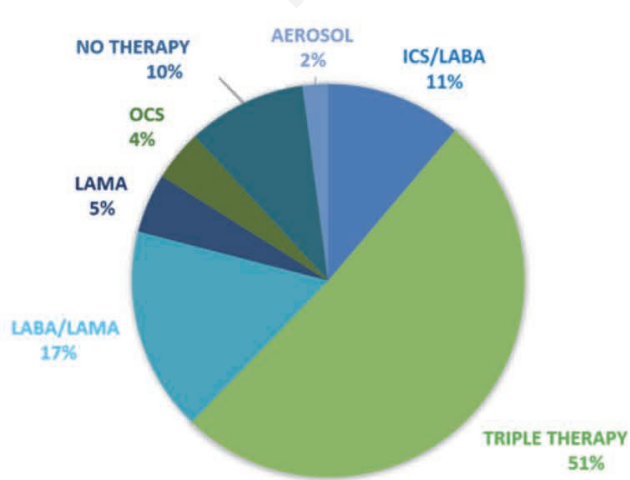

Figure 1. Treatment for chronic obstructive pulmonary disease at discharge, according to GOLD class. ICS, inhaled corticosteroid; $L A B A$, long-acting $\beta$-adrenoceptor agonist; LAMA, long-acting muscarinic agonist; OCS, an oral corticosteroid.

\section{Discussion and Conclusions}

Few data are available in the literature regarding the diagnostic and therapeutic pathway of patients with exacerbation of COPD immediately after hospitalization. ${ }^{10}$ The key objectives of this study were to provide a reliable profile of COPD patients admitted to IMUs in terms of clinical presentation, comorbidities, and history of exacerbations, as well as a picture of the types of treatments used by Internists for COPD. Further, we tested a post-discharge monitoring strategy in order to evaluate the applicability of some management tools (such as dedicated reports and discharge checklists, as well as follow-up sheets created for that purpose and disease-specific ${ }^{7}$ ), used systematically in a large number of Italian IMUs.

In general, the phenotype of the patient hospitalized in Internal Medicine for exacerbation of COPD is of an older person, predominantly male, usually with a known diagnosis of COPD and a former or current smoker, with predominantly cardiovascular or metabolic comorbidities, with dyspnoea and respiratory failure as the main clinical manifestations at admission, and usually with a history of 1-2 exacerbations/year and in most cases with mild-mod-

Table 2. Main indications delivered at discharge through the follow-up sheet used in the study.

\begin{tabular}{lc}
\hline Indication & $\begin{array}{c}\text { General study } \\
\text { population (n=472) }\end{array}$ \\
\hline Planned duration of follow-up & $152(32.2 \%)$ \\
- 1 month & $135(28.6 \%)$ \\
- 2-3 months & $129(27.3 \%)$ \\
- 4-6 months & $11(2.3 \%)$ \\
- 9 months & $45(9.5 \%)$ \\
- 12 months & \\
\hline Clinical control & $191(40.5 \%)$ \\
- General Practitioner & $152(32.2 \%)$ \\
- Specialist & $129(27.3 \%)$ \\
- Self-monitoring & \\
\hline Chest X-ray & $56(11.9 \%)$ \\
- 1-3 months & $52(11.0 \%)$ \\
- 4-6 months & $29(6.1 \%)$ \\
- 12 months & $335(71.0 \%)$ \\
- Not necessary & \\
\hline Spirometry & $123(26.1 \%)$ \\
- 1-3 months & $99(21.0 \%)$ \\
- 4-6 months & $56(11.9 \%)$ \\
- 12 months & $194(41.0 \%)$ \\
\hline - Not necessary & \\
\hline Blood gas analysis & $128(27.1 \%)$ \\
- 1-3 months & $99(21.0 \%)$ \\
- 4-6 months & $19(4.0 \%)$ \\
- 12 months & $226(47.9 \%)$ \\
\hline Not necessary & \\
Monitoring of comorbidities & $93(19.7 \%)$ \\
- Disease 1 & $60(12.7 \%)$ \\
- Disease 2 & $18(3.8 \%)$ \\
\hline Disease 3 &
\end{tabular}


erate symptoms in the stable phase of the disease.

As previously reported, it is undoubtedly important to reflect on discharge treatments concerning GOLD class, ${ }^{11}$ and this is confirmed according to our data. Virtually all treatment options are in stage A, with dual or triple therapy in approximately $50 \%$ of cases, which rises to $60 \%$ in stage B. In stage C, we should consider that approximately $15 \%$ of patients have no therapy or oral steroids. Finally, triple therapy is present in $50 \%$ of stage $\mathrm{D}$ patients, and also in this case, $10 \%$ of patients are without therapy and $4 \%$ are treated with oral steroids.

One of the main objectives of the study was to focus on the time when the patient is discharged from hospital, in order to promote systematic prevention of exacerbations and re-hospitalizations. ${ }^{12}$ To this end, the Internist can and should use therapeutic algorithms, minimizing the risk of inappropriateness, suggesting more beneficial treatments for the patient, and facilitating the application of simplified (and therefore more viable) and more homogeneous behaviors. This is also to take advantage of the fact that we currently have COPD treatments that can reduce hospitalizations and mortality by differentiating the drugs used based on the characteristics of the individual patient. ${ }^{13}$ In order to optimize the effects of treatments, the proper utilization of devices and the effective control of therapeutic adherence are crucial. ${ }^{14}$

The follow-up sheet used in our study was shown as applicable. It was used to define the modality of clinical control after discharge and the people who performed it, and to assess the appropriateness and timing of subsequent tests, for example in terms of blood gas reassessment; in $48 \%$ of cases, the latter was deemed unnecessary, even though with some physiological doubts. It should be noted that the proportion of patients for whom a medical check is considered necessary is quite high $(73 \%)$, but the indication to perform a subsequent chest X-ray occurred in $29 \%$ of cases; the spirometry test was not prescribed in $41 \%$ of patients for up to 1 year. The proportion of patients for whom a check was deemed necessary within a brief period of time was quite high ( $61 \%$ of patients within 3 months). Finally, it is not very encouraging to see that the activation of lon- gitudinal checks for comorbidities was only scheduled for $19 \%$ of patients, and this despite established evidence of the weight and role of comorbidities in the case of a COPD patient admitted to Internal Medicine. ${ }^{15}$ These indications, as much as they can be discussed and interpreted in different ways, still represent a snapshot of the real world, which is one of the strengths of this project, providing useful insights for education and awareness.

In conclusion, the study has allowed us to have a nationwide description of the characteristics of patients with COPD exacerbation admitted to IMUs, and of the treatments prescribed according to the GOLD criteria. The results confirmed the multimorbidity and complexity that characterize patients with this disease, and they have allowed us to document how treatment options are used. Although it analyzed a relatively small cohort of patients, the study also reported what would be the main indications for postdischarge management of the patient with COPD. This has highlighted the need for greater awareness and more standardized procedures within IMUs for the management of patients with COPD, especially in the post-discharge phase. In this perspective, our findings seem to encourage greater focus on some COPD-specific diagnostic and monitoring procedures, which could also be useful for better management of frequently occurring comorbidities. All of the above is more COPD patient-oriented rather than something simply aimed at treating the disease. This process may also be pursued further through the adoption of COPD-specific documentation, including a dedicated report and discharge checklist, and a follow-up sheet like those used and tested in the study. These might allow better patient monitoring, by establishing a communication channel with the local health services for outpatients, and greater involvement and accountability of the patient/caregiver. In the future, such documentation may be integrated into a comprehensive model including address lines for inhaled therapy and, more generally, for optimized home management, to be disseminated at IMUs through structured training pathways (e.g. outreach visits) that also include longitudinal analyses of procedural and outcome improvements.

\section{APPENDIX I}

Specific Hospital Discharge Form for the patient with exacerbation of COPD discharged from Internal Medicine Units (IMUs) (available online). 


\section{APPENDIX II}

We would like to mention and thank all the members of the SDO-ARCA Study Group who actively contributed in the enrolment of patients and data collection (listed per center and according to the number of enrolled patients):

M. Candela, M. Onesta, (Fabriano - AN); S. Marengo, C. Norbiato (Torino); M. Bigliocca, M. Campanini, E. Catania, E. Maggi (Novara); A. Brucato, A. Cammà (Bergamo); R. Pastorelli, S. Di Simone (Colleferro RM); R. Frediani, A. Moschella (Verbania - VCO); G. Panigada, L. Teghini, R. Pierotello, L. Venturini (Pescia - PT); L. Rasciti, M.L. Cipollini, E. Sagrini (Ravenna); A. Benvenuto, A.M. Carella (San Severo - FG); L. Anastasio (Vibo Valentia); E. Leva (Acerra - NA); L. Muraca, L. Ammendola, G. Putortì, C. Madeo, P. Salerno (Corigliano Calabro - CS); L. Magnani, P. Cavallo, A. Gallia (Voghera - PV); F. Corradi, S. Fruttuoso (Firenze); L. Vendemiale, C. Capurso (Foggia); L.E. Adinolfi, A. Santoro (Marcianise - CE); E. Chessa (Oristano); R. Risicato, E. Cristaldi (Augusta - SR); L. Procopio, A. Raco, S. Pavano (Menaggio - CO); D. Manfellotto, P. Anselmi, G. B. Capoccetta (Roma); L. Morbidoni, S. Arduino (Senigallia - AN); F. Nasso, R. M. Macchione, V. Gioffrè (Polistena - RC);V. Catania, G. Di Vita (Gela - CL); M. Di Natale, P. Lotti, S. Zanieri (Prato); V. Provenzano, D. Brancato, M.L. Lo Cascio (Partinico - PA); F. Colombo, A. Carbone (Milano); S. Ballestri, F. Lami (Pavullo nel Frignano - MO); A. Salvi, C. Nitti (Ancona); M. Favro, V. Tomaselli (Asti); A. Puleo, C. Lucia (Palermo); F. Sdanganelli (Taranto); S. De Carli (Udine); M.C. Bertoncelli, C. Manfrinato (Vercelli); M. Marvisi, S. Ramponi (Cremona); P. Ghiringhelli, P. Novati (Saronno - VA); A. Fontanella, M. Poggiano (Napoli); M. Fioretti (Terni); G. Zavarise (Recco - GE); A. Sacchetta, A. Vendrame; S. Guzzon (Conegliano Veneto - TV); A. Mazzone, G. Bonardi (Legnano - MI); A. Zuccoli (NA); G. Battaglia, G. Ciarallo (Santorso - VI).

\section{References}

1. Lozano R, Naghavi M, Foreman K, et al. Global and regional mortality from 235 causes of death for 20 age groups in 1990 and 2010: a systematic analysis for the Global Burden of Disease Study 2010. Lancet 2012;380: 2095-128.

2. Mathers CD, Loncar D. Projections of global mortality and burden of disease from 2002 to 2030 . PLoS Med 2006;3:e442.

3. Vanfleteren LEGW, Spruit MA, Groene M, et al. Clusters of comorbidities based on validated objective measurements and systemic inflammation in patients with chronic obstructive pulmonary disease. Am J Respir Crit Care Med 2013;187:728-35.

4. Global Initiative for Chronic Obstructive Lung Disease 2019. Eur Respir J 2019;53:1900164.

5. Pedersen PU, Ersgard KB, Soerensen TB, Larsen P. Effectiveness of structured planned post discharge support to patients with chronic obstructive pulmonary disease for reducing readmission rates: a systematic review. JBI Database System Rev Implement Rep 2017;15:2060-86.

6. Jordan RE, Majothi S, Heneghan NR, et al. Supported self-management for patients with moderate to severe chronic obstructive pulmonary disease (COPD): an evidence synthesis and economic analysis. Health Technol Assess 2015;19:1-516.

7. Majothi S, Jolly K, Heneghan NR, et al. Supported selfmanagement for patients with COPD who have recently been discharged from hospital: a systematic review and meta-analysis. Int J Chron Obstruct Pulmon Dis 2015; 10:853-67.

8. López-Campos JL, Quintana Gallego E, Carrasco
Hernández L. Status of and strategies for improving adherence to COPD treatment. Int J Chron Obstruct Pulmon Dis 2019;14:1503-15.

9. Candela M, Pastorelli R, Sacchetta A, et al. Management of chronic obstructive pulmonary disease: criteria for an appropriate hospital discharge. Ital $\mathrm{J}$ Med 2013;7:218-30.

10. Adamson SL, Burns J, Camp PG, et al. Impact of individualized care on readmissions after a hospitalization for acute exacerbation of COPD. Int J Chron Obstruct Pulmon Dis 2016;11:61-71.

11. David M, Halpin G, de Jong HJI, et al. Distribution, temporal stability and appropriateness of therapy of patients with COPD in the UK in relation to GOLD 2019. E Clin Med 2019;14:32-41.

12. Press VG, Au DH, Bourbeau J, et al.; on behalf of the American Thoracic Society Behavioral Science and Health Services Research, Clinical Problems, and Nursing Assemblies. Reducing Chronic Obstructive Pulmonary Disease Hospital Readmissions. An Official American Thoracic Society Workshop Report. Annals ATS 2019;16:2.

13. Lipson DA, Barnhart F, Brealey N, et al.; for the IMPACT Investigators. Once-Daily Single-Inhaler Triple versus Dual Therapy in Patients with COPD. N Engl J Med 2018;378;1671-80.

14. Vestbo J, Anderson A, Calverley PMA, et al. Adherence to inhaled therapy, mortality and hospital admission in COPD. Thorax 2009;64:939-43.

15. Nozzoli C, Anastasio L, Fabbri LM, et al.; for the Research Department of FADOI. Complexity of patients with chronic obstructive pulmonary disease hospitalized in internal medicine: a survey by FADOI. Ital J Med 2015;9:120-4. 